\title{
Quantum Metrology with a Scanning Probe Atom Interferometer
}

\author{
Caspar F. Ockeloen, Roman Schmied, Max F. Riedel, and Philipp Treutlein* \\ Department of Physics, University of Basel, Klingelbergstrasse 82, 4056 Basel, Switzerland
}

(Received 6 March 2013; published 3 October 2013)

\begin{abstract}
We use a small Bose-Einstein condensate on an atom chip as an interferometric scanning probe to map out a microwave field near the chip surface with a few micrometers resolution. With the use of entanglement between the atoms, our interferometer overcomes the standard quantum limit of interferometry by $4 \mathrm{~dB}$ and maintains enhanced performance for interrogation times up to $10 \mathrm{~ms}$. This corresponds to a microwave magnetic field sensitivity of $77 \mathrm{pT} / \sqrt{\mathrm{Hz}}$ in a probe volume of $20 \mu \mathrm{m}^{3}$. Quantum metrology with entangled atoms is useful in measurements with high spatial resolution, since the atom number in the probe volume is limited by collisional loss. High-resolution measurements of microwave near fields, as demonstrated here, are important for the development of integrated microwave circuits for quantum information processing and applications in communication technology.
\end{abstract}

DOI: 10.1103/PhysRevLett.111.143001

Interferometers operating with large atomic ensembles offer unsurpassed precision in measurements of inertial forces, atomic properties, and fundamental constants and currently define the standard of time [1,2]. Using a small atomic cloud as a scanning probe interferometer with high spatial resolution would enable new applications in electromagnetic field sensing, surface science, and the search for fundamental short-range interactions [1]. However, as a small probe necessarily contains only a small number of atoms, the standard quantum limit (SQL) due to projection noise [3] places a tight bound on the achievable precision. We report a scanning probe atom interferometer that overcomes the SQL by entangling the atoms and use it for a high-resolution microwave field measurement. With a spin-squeezed Bose-Einstein condensate [4,5] (BEC) on an atom chip [6], we achieve a precision of $4 \mathrm{~dB}$ in variance (a factor 1.6 in standard deviation) below the SQL. Performance below the SQL is maintained for interrogation times up to $10 \mathrm{~ms}$, more than an order of magnitude longer than that in previous experiments $[5,7,8]$. We transport our $1.1-\mu \mathrm{m}$-sized probe between 40 and $16 \mu \mathrm{m}$ from the atom chip surface and record the spatial profile of a microwave magnetic field with sub-SQL precision. These techniques are relevant for the high-resolution imaging of electromagnetic fields near microfabricated structures [9-13].

In an atom interferometer, the external (motional) or internal (spin) state of atoms is coherently split and allowed to follow two different pathways [1,2]. During the interrogation time $T_{R}$, a phase $\varphi$ between the paths is accumulated, which depends on the quantity to be measured. When the paths are recombined, the wave character

Published by the American Physical Society under the terms of the Creative Commons Attribution 3.0 License. Further distribution of this work must maintain attribution to the author(s) and the published article's title, journal citation, and DOI.
PACS numbers: 37.25.+k, 03.75.Gg, 06.20.-f, 42.50.Lc

of the atoms gives rise to an interference pattern, from which $\varphi$ can be determined. In recording this interference, however, the particle character of the atoms is revealed, as a measurement randomly projects the wave function of each atom into a definite state. When operating with an ensemble of $N$ uncorrelated (nonentangled) atoms, the resulting binomial statistics of counting individual atoms in the output states limits the phase uncertainty of the interferometer to $\sigma_{\varphi} \geq 1 / \sqrt{N}$, the standard quantum limit (SQL) of interferometric measurement [3].

An obvious way to reduce $\sigma_{\varphi}$ is to increase the number of particles in the interferometer. Most atom interferometers thus operate with large ensembles containing millions of atoms. However, there are important cases where a physical process places an upper bound on $N$, so that this strategy is not feasible. In chip-based atomic clocks, for example, undesired collisional frequency shifts limit the usable atomic density and thus the atom number to $N \lesssim$ $10^{5}$ [14]. A particularly tight limit applies to atom interferometry with high spatial resolution, where the required small probe size gives rise to an upper bound on $N$ due to density-dependent collisional trap losses. In our experiment, operating with ${ }^{87} \mathrm{Rb}$ atoms in a probe of $20 \mu \mathrm{m}^{3}$ volume, inelastic two-body collisions result in a limit of $N \lesssim 10^{5}$ for $T_{R}=10 \mathrm{~ms}$, or $N \lesssim 10^{3}$ for $T_{R}=1 \mathrm{~s}$. Increasing $N$ beyond these limits yields no further improvement in precision, since the additional particles are quickly lost from the trap [Supplemental Material [15]]. Similar limits apply to interferometers with other atomic species.

Quantum metrology provides a way to overcome the SQL at fixed $N$ by using entanglement between the probe particles [16]. This allows reducing the phase measurement uncertainty towards the ultimate Heisenberg limit $\sigma_{\varphi} \geq$ $1 / N$. An important class of interferometrically useful entanglement is provided by spin-squeezed states $[17,18]$, characterized by the squeezing parameter [19] $\xi<1$, defined such that $\sigma_{\varphi}=\xi / \sqrt{N}$. Recent experiments with 
atomic ensembles demonstrated suitable entangled states [4,20-25]. With optically trapped atoms, full interferometer sequences were implemented [5,7,8], with a focus on atomic clocks and measurements of homogeneous magnetic fields $[26,27]$. These experiments achieve improvements of up to $\xi^{2}=-4 \mathrm{~dB}$ in variance over the SQL for interrogation times up to $T_{R}=600 \mu \mathrm{s}$ [8]. This is still far from the Heisenberg limit, and substantial improvements in $\xi^{2}$ and $T_{R}$ are desired.

For scanning probe measurements near a surface, magnetic traps on atom chips [6] have proven to be useful, as they provide submicrometer position control over small BECs [9-11,13,28,29]. Such systems have long been considered for quantum metrology, but up to now neither an interferometric scanning probe nor a measurement beyond the SQL had been demonstrated.

Our interferometric scanning probe is a ${ }^{87} \mathrm{Rb}$ BEC prepared close to the surface of an atom chip. The two ground-state hyperfine levels $|1\rangle=\left|F=1, m_{F}=-1\right\rangle$ and $|2\rangle=\left|F=2, m_{F}=1\right\rangle$ serve as interferometer pathways. Figure 1 shows an overview of our experimental setup $[4,15]$. The atom chip has two layers of wires carrying both continuous (dc) and microwave (mw) currents. The dc currents generate magnetic traps, which are identical for both states. In each run of the experiment, we start by preparing a pure BEC of $N=1400 \pm 40$ atoms (shot-to-shot preparation noise) in state $|1\rangle$, in a trap situated $40 \mu \mathrm{m}$ from the chip surface. This trap can be smoothly shifted towards the chip surface to perform high-resolution measurements. The probe size is characterized by the BEC radii of $R_{y}=R_{z}=1.1 \mu \mathrm{m}$ and $R_{x}=4.0 \mu \mathrm{m}$ [Supplemental Material [15]].

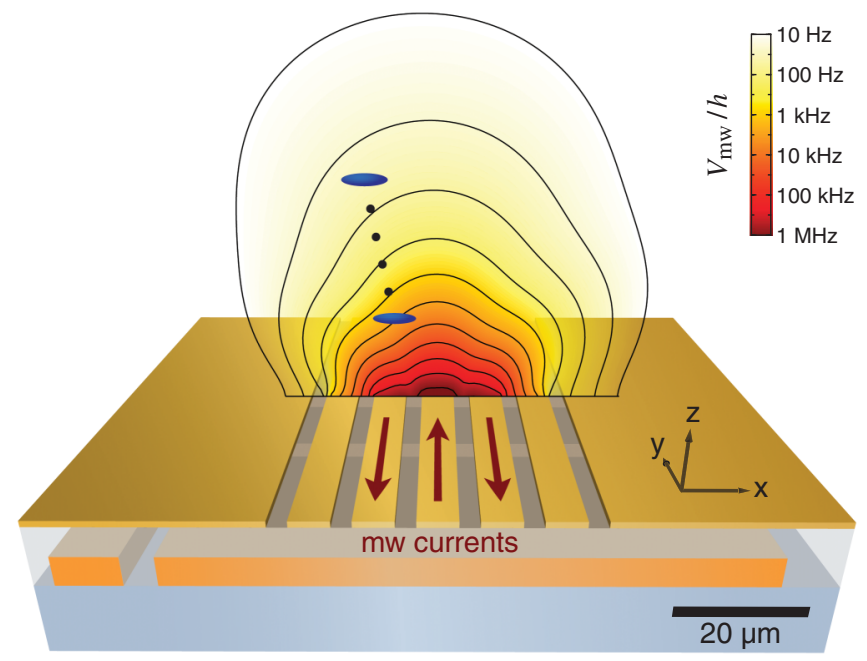

FIG. 1 (color). Experimental setup. Central region of the atom chip showing the atomic probe (blue, size to scale) and the scanning trajectory we use (between 40 and $16 \mu \mathrm{m}$ from the chip surface). The probe is used to measure the magnetic nearfield potential generated by an on-chip microwave guide (microwave currents indicated by arrows). A simulation of the potential is shown in red and yellow.
To create entanglement between the atoms, we make use of two-body collisions. Normally, these collisions have negligible effect on the internal state of the BEC, due to a coincidence of the scattering lengths of our state pair. A crucial feature of our experiment is that we can "turn on" the effect of collisions for a well-defined time by spatially separating the states [30]. For this we use a state-dependent potential, which is generated by the on-chip microwave currents. By recombination of the states, the collisions are effectively "turned off" again so that they do not perturb the subsequent interferometric sequence. In Ref. [4] we used this technique to produce spin-squeezed states of a BEC.

The internal state of our BEC can be described by a collective spin $\vec{S}$, whose quantum state is visualized on a sphere [Fig. 2(a)]. The $S_{z}$ component is proportional to the population difference $n=\left(N_{1}-N_{2}\right) /\left(N_{1}+N_{2}\right)$, where $N_{i}$ is the atom number in state $|i\rangle$. The azimuthal angle is the relative phase $\varphi$ between $|1\rangle$ and $|2\rangle$. Using mw and radio-frequency (rf) Rabi pulses generated off chip, we can

(a)
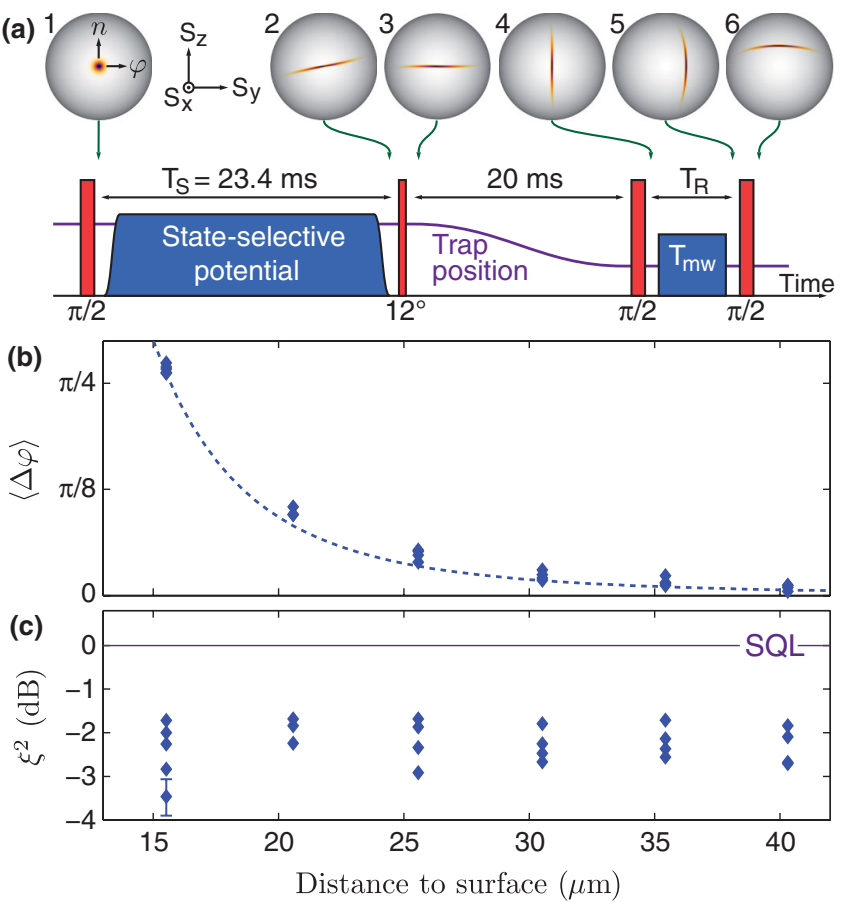

FIG. 2 (color). Scanning probe interferometer operating below the SQL. (a) Experimental sequence, showing Rabi (red) and on-chip microwave (blue) pulses and the trap position (purple). Spheres 1-6 show the Wigner function [39] of the collective spin state at various stages of the experiment, simulated for $N=200$ atoms. (b) Measured phase shift $\langle\Delta \varphi\rangle$ induced by a microwave near-field pulse as a function of the atom-surface distance, compared to the simulated potential (dotted line, see also Fig. 1). (c) Measured performance of the interferometer, expressed as squeezing factor $\xi^{2}$. Each data point (based on 240 measurements) has a statistical uncertainty of $\pm 0.4 \mathrm{~dB}$, shown as an error bar on the lower left point. The experiment was repeated up to 5 times at each position. 
coherently couple $|1\rangle$ and $|2\rangle$, thereby rotating the state on the sphere. The effect of collisions is well described by the Hamiltonian $H_{\text {int }}=\hbar \chi S_{z}^{2}$, which creates spin squeezing by "twisting" the state on the sphere $[17,18,30]$. The rate $\chi$ is controlled by the state-dependent potential as described above.

The experimental sequence of our scanning probe interferometer is shown in Fig. 2(a). We start with a $\pi / 2$ pulse, creating a coherent spin state (sphere 1) on the equator of the sphere. Then, we apply $H_{\text {int }}$ for $T_{\mathrm{S}}=23.4 \mathrm{~ms}$ by turning on the state-dependent potential. The result is a spin-squeezed state (sphere 2) with a measured $\xi^{2}=$ $-4.3 \pm 0.4 \mathrm{~dB}$ (statistical) $\pm 0.4 \mathrm{~dB}$ (systematic). The systematic uncertainty arises from calibrating the imaging system and applies to all measurements of $\xi^{2}$ in this Letter [Supplemental Material [15]]. Next, we apply a $-12^{\circ}$ rotation around the $S_{x}$ axis, aligning the antisqueezed quadrature with the equator (sphere 3). This renders the state minimally sensitive to phase noise during the following $20 \mathrm{~ms}$, in which we transport the atoms to the position where the interferometric measurement is to be made [Supplemental Material [15]]. Finally, we perform a full Ramsey interferometer sequence consisting of a $\pi / 2$ pulse around the state's center to make it maximally phase sensitive (sphere 4), an evolution time $T_{R}$ during which the phase $\varphi$ is accumulated (sphere 5), and a final $\pi / 2$-pulse mapping $\varphi$ onto $n$ (sphere 6). We read out the population difference, which oscillates as $n=$ $C \sin \varphi$, where $C \leq 1$ is the interferometric contrast. Experimentally, an offset phase $\theta$ can be added to the last $\pi / 2$ pulse. For example, we can choose $\theta$ such that $\langle n\rangle=0$ and the interferometric phase uncertainty is $\sigma_{\varphi}=\sigma_{n} / C$, where $\sigma$ denotes standard deviation.

We demonstrate the scanning probe interferometer with spatially resolved measurements of the on-chip microwave near field at $6.8 \mathrm{GHz}$. We use $T_{R}=100 \mu \mathrm{s}$, during which we pulse on the field for $T_{\mathrm{mw}}=80 \mu \mathrm{s}$ with a detuning of $12 \mathrm{MHz}$ above the $\left|F=1, m_{F}=0\right\rangle \rightarrow\left|F=2, m_{F}=0\right\rangle$ transition. This results in an additional phase shift $\Delta \varphi=$ $T_{\mathrm{mw}} V_{\mathrm{mw}} / \hbar$, where $V_{\mathrm{mw}}$ is the differential ac Zeeman shift of states $|1\rangle$ and $|2\rangle$ [Supplemental Material [15]]. Figure 2(b) shows measurements at the positions indicated in Fig. 1, between 40 and $16 \mu \mathrm{m}$ from the chip surface. At each position we measure $\varphi$ and the reference phase $\varphi_{0}$ in a separate measurement without mw pulse. The mean phase shift due to the mw is given by $\langle\Delta \varphi\rangle=\langle\varphi\rangle-\left\langle\varphi_{0}\right\rangle$. We extract these mean phases from fits of sine functions to the measured values of $n$ as a function of $\theta$. The measured shape of $V_{\mathrm{mw}}$ agrees well with a simulation based on previous measurements using a different technique [12]. The present interferometric measurement is more precise and offers higher spatial resolution. Figure 2(c) shows the performance of our interferometer in terms of the squeezing factor $\xi^{2}$, measured while operating at $\langle n\rangle=0$ with $\mathrm{mw}$ pulse on. For all positions, our interferometer performs well below the SQL, with an average performance of $\left\langle\xi^{2}\right\rangle=$ $-2.2 \mathrm{~dB}$ corresponding to a single-shot phase sensitivity of $\sigma_{\varphi}=1.2^{\circ}$, or a microwave field sensitivity of $\delta B=$ $2.4 \mu \mathrm{T}$. We attribute the reduction of squeezing compared to the input state to the extra Rabi pulses and extra time needed to transport the atoms.

In high-resolution atom interferometry, several effects impose a limit on probe size and interrogation time. To resolve the spatial features of the microwave near field, the probe size has to be much smaller than the characteristic size of the field generating structure $(\approx 10 \mu \mathrm{m}$ in our case) and the probe-surface distance. Closely related, the gradients of the field across the probe lead to inhomogeneous dephasing, reducing $C$, and imposing a limit on the product $T_{\mathrm{mw}} V_{\mathrm{mw}}$. Furthermore, $T_{\mathrm{mw}}$ must be sufficiently short to avoid demixing of states $|1\rangle$ and $|2\rangle$. In our experiment, we choose $T_{\mathrm{mw}} V_{\mathrm{mw}}$ such that the contrast at $16 \mu \mathrm{m}$ from the surface is still $C \geqslant 95 \%$.

For small $V_{\mathrm{mw}}$, interferometric sensitivity scales linearly with $T_{R}$, and long interrogation times are desired. We therefore measure the performance of our interferometer as a function of $T_{R}$, similar to the case in Ref. [8]. We use the same sequence as described above, but omit transporting the atoms. Instead, the second and third Rabi pulses are combined into a single $+78^{\circ}$ rotation, which aligns the squeezed quadrature with the equator and immediately starts the free evolution. During $T_{R}$, no additional phase shift is applied.

Figure 3 shows the measured phase uncertainty for varying $T_{R}$ values. The interferometer operates at $\xi^{2} \approx$ $-4 \mathrm{~dB}$ below the SQL, maintaining the squeezing level of

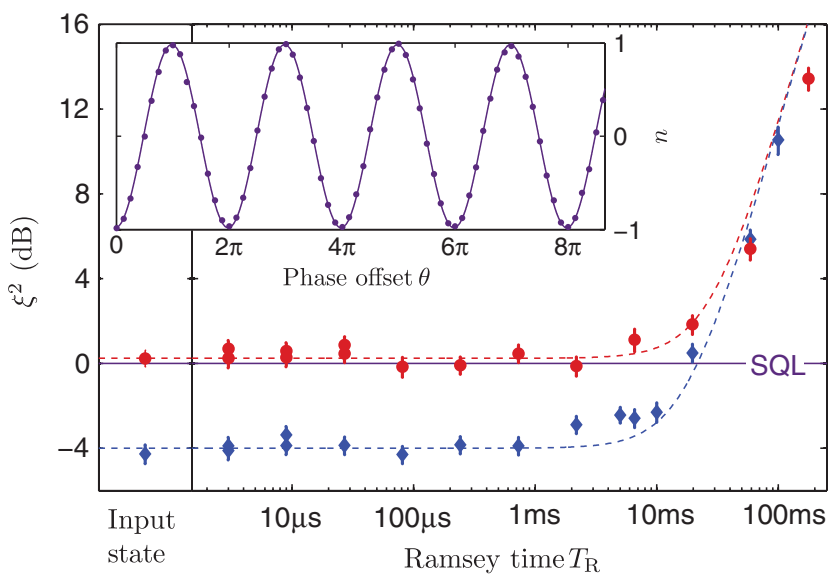

FIG. 3 (color). Interferometer performance. Observed phase noise in a Ramsey interferometer with squeezed (blue diamonds) and coherent (red circles) input states for varying interrogation times $T_{R}$. Dashed lines model constant performance of $\xi^{2}=$ $-4 \mathrm{~dB}$ (squeezed state) and $+0.2 \mathrm{~dB}$ (coherent state), plus technical noise due to shot-to-shot frequency fluctuations. Each data point is the result of 240 measurements, and error bars indicate statistical uncertainty. Inset: typical squeezed-state Ramsey fringe measurements for $T_{R}=5 \mathrm{~ms}$ (points) and fitted sine (line) yielding a contrast of $C=(98.1 \pm 0.2) \%$. 
the input state. It performs better than the SQL up to $T_{R}=$ $10 \mathrm{~ms}$, an improvement by a factor of 17 compared to that of previous results [8]. In a reference measurement using a coherent state, our performance is consistent with the SQL plus independently measured detection noise of $\sigma_{n \text {,det }}=$ $5.1 \times 10^{-3}$ (5 times smaller than the SQL). After $T_{R}>$ $20 \mathrm{~ms}$, both measurements are limited by technical noise, consistent with shot-to-shot fluctuations of $150 \mathrm{mHz}$ (rms) in the relative frequency between our reference oscillator and the atomic resonance.

High-resolution measurements of microwave near fields are relevant for scientific applications, e.g., in superconducting quantum information processing [31] and for the design of new microwave circuits for use in communication technology [32]. A standard technique for the imaging of microwave fields does not exist, and a technique based on atom interferometry has the advantage of being intrinsically calibrated. To fully characterize microwave near fields, both the electric and magnetic field component have to be measured. In recent proof-of-principle experiments, the electric field was detected using Rydberg atoms [33], and the magnetic field components were imaged using ground-state atoms and a noninterferometric scheme $[12,34]$. The entanglement-enhanced interferometric sensor demonstrated here is about 3 orders of magnitude more sensitive to the magnetic field component and offers higher spatial resolution.

At $T_{R}=10 \mathrm{~ms}$, our measured noise level corresponds to a single-shot sensitivity in the ac Zeeman shift of $\delta V_{\mathrm{mw}} / h=0.33 \mathrm{~Hz}$. We estimate the corresponding microwave field sensitivity for a near-resonant field, obtaining a noise-equivalent field amplitude of $\delta B \approx \delta V_{\mathrm{mw}} / \mu_{B}=$ $23 \mathrm{pT}$. Taking our experimental cycle of $11 \mathrm{~s}$ into account, we obtain a microwave field sensitivity of $77 \mathrm{pT} / \sqrt{\mathrm{Hz}}$. We emphasize that this sensitivity is achieved with a small probe of $20 \mu \mathrm{m}^{3}$ in volume. Room-temperature vapor cell magnetometers achieve subfemtotesla sensitivity in the measurement of static and rf fields [35], operating with probe volumes of cubic millimeters [36] to centimeters [26]. In their present form, they cannot resolve fields on the micrometer scale, such as the near field of the chip in our experiment. When scaled down to micrometer size, the number of atoms in the probe volume decreases, lowering the sensitivity. Assuming projection-noise scaling, a stateof-the-art vapor cell magnetometer such as in Refs. [26,36] but with a probe volume comparable to our BEC would have a sensitivity of $300 \mathrm{pT} / \sqrt{\mathrm{Hz}}$. This shows that our experiment achieves an excellent field sensitivity at the micrometer scale. Comparable sensitivity has been achieved with cold atom sensors that provide differential measurements of static magnetic fields [28,37]. In contrast, our interferometric sensor performs an absolute measurement of a microwave field amplitude. Finally, we point out that nitrogen vacancy centers in diamond are outstanding magnetic field sensors at the nanometer scale; however, at micrometer resolution the sensitivities are lower than those demonstrated with atoms [38].

In conclusion, we have experimentally demonstrated a scanning probe atom interferometer operating beyond the standard quantum limit and used it for the measurement of a microwave near field. This is the first demonstration of entanglement-enhanced atom interferometry with a high spatial resolution scanning probe and promises further high-resolution sensing and measurement applications.

We thank P. Böhi for help with the experimental setup. This work was supported by the Swiss National Science Foundation and by the EU projects AQUTE and QIBEC.

*philipp.treutlein@unibas.ch

[1] A. D. Cronin, J. Schmiedmayer, and D. E. Pritchard, Rev. Mod. Phys. 81, 1051 (2009).

[2] J. Kitching, S. Knappe, and E. A. Donley, IEEE Sens. J. 11, 1749 (2011).

[3] W. Itano, J. Bergquist, J. Bollinger, J. Gilligan, D. Heinzen, F. Moore, M. Raizen, and D. Wineland, Phys. Rev. A 47, 3554 (1993).

[4] M. F. Riedel, P. Böhi, Y. Li, T. W. Hänsch, A. Sinatra, and P. Treutlein, Nature (London) 464, 1170 (2010).

[5] C. Gross, T. Zibold, E. Nicklas, J. Estève, and M. K. Oberthaler, Nature (London) 464, 1165 (2010).

[6] Atom Chips, edited by J. Reichel and V. Vuletić (WileyVCH, Weinheim, Germany, 2011).

[7] A. Louchet-Chauvet, J. Appel, J. J. Renema, D. Oblak, N. Kjaergaard, and E.S. Polzik, New J. Phys. 12, 065032 (2010).

[8] I. D. Leroux, M. H. Schleier-Smith, and V. Vuletić, Phys. Rev. Lett. 104, 250801 (2010).

[9] Y.-j. Lin, I. Teper, C. Chin, and V. Vuletić, Phys. Rev. Lett. 92, 050404 (2004).

[10] J. M. Obrecht, R. J. Wild, and E. A. Cornell, Phys. Rev. A 75, 062903 (2007).

[11] S. Aigner, L. Della Pietra, Y. Japha, O. Entin-Wohlman, T. David, R. Salem, R. Folman, and J. Schmiedmayer, Science 319, 1226 (2008).

[12] P. Böhi, M. F. Riedel, T. W. Hänsch, and P. Treutlein, Appl. Phys. Lett. 97, 051101 (2010).

[13] M. Gierling, P. Schneeweiss, G. Visanescu, P. Federsel, M. Häffner, D. P. Kern, T. E. Judd, A. Günther, and J. Fortágh, Nat. Nanotechnol. 6, 446 (2011).

[14] P. Rosenbusch, Appl. Phys. B 95, 227 (2009).

[15] See the Supplemental Material at http://link.aps.org/ supplemental/10.1103/PhysRevLett.111.143001 for detailed description of the experimental setup, collisional trap loss, the state-dependent microwave potential, atom number detection, and data analysis.

[16] V. Giovannetti, S. Lloyd, and L. Maccone, Nat. Photonics 5, 222 (2011).

[17] M. Kitagawa and M. Ueda, Phys. Rev. A 47, 5138 (1993).

[18] A. Sørensen, L.-M. Duan, J. I. Cirac, and P. Zoller, Nature (London) 409, 63 (2001). 
[19] D. J. Wineland, J. J. Bollinger, W. M. Itano, and D. J. Heinzen, Phys. Rev. A 50, 67 (1994).

[20] J. Estève, C. Gross, A. Weller, S. Giovanazzi, and M. K. Oberthaler, Nature (London) 455, 1216 (2008).

[21] J. Appel, P. J. Windpassinger, D. Oblak, U. B. Hoff, N. Kjaergaard, and E. S. Polzik, Proc. Natl. Acad. Sci. U.S.A. 106, 10960 (2009).

[22] M. H. Schleier-Smith, I. D. Leroux, and V. Vuletić, Phys. Rev. Lett. 104, 073604 (2010).

[23] B. Lücke et al., Science 334, 773 (2011).

[24] C. Gross, H. Strobel, E. Nicklas, T. Zibold, N. Bar-Gill, G. Kurizki, and M. K. Oberthaler, Nature (London) 480, 219 (2011).

[25] C.D. Hamley, C.S. Gerving, T.M. Hoang, E. M. Bookjans, and M. S. Chapman, Nat. Phys. 8, 305 (2012).

[26] W. Wasilewski, K. Jensen, H. Krauter, J. J. Renema, M. V. Balabas, and E. S. Polzik, Phys. Rev. Lett. 104, 133601 (2010).

[27] R. J. Sewell, M. Koschorreck, M. Napolitano, B. Dubost, N. Behbood, and M. W. Mitchell, Phys. Rev. Lett. 109, 253605 (2012).

[28] S. Wildermuth, S. Hofferberth, I. Lesanovsky, S. Groth, P. Krüger, J. Schmiedmayer, and I. Bar-Joseph, Appl. Phys. Lett. 88, 264103 (2006).
[29] D. Hunger, S. Camerer, T. W. Hänsch, D. König, J. P. Kotthaus, J. Reichel, and P. Treutlein, Phys. Rev. Lett. 104, 143002 (2010).

[30] Y. Li, P. Treutlein, J. Reichel, and A. Sinatra, Eur. Phys. J. B 68, 365 (2009).

[31] S. D. Hogan, J. A. Agner, F. Merkt, T. Thiele, S. Filipp, and A. Wallraff, Phys. Rev. Lett. 108, 063004 (2012).

[32] S. Sayil, D. Kerns, and S. Kerns, IEEE Trans. Instrum. Meas. 54, 2082 (2005).

[33] J. A. Sedlacek, A. Schwettmann, H. Kübler, R. Löw, T. Pfau, and J. P. Shaffer, Nat. Phys. 8, 819 (2012).

[34] P. Böhi and P. Treutlein, Appl. Phys. Lett. 101, 181107 (2012).

[35] D. Budker and M. Romalis, Nat. Phys. 3, 227 (2007).

[36] R. Mhaskar, S. Knappe, and J. Kitching, Appl. Phys. Lett. 101, 241105 (2012).

[37] M. Vengalattore, J. M. Higbie, S. R. Leslie, J. Guzman, L. E. Sadler, and D. M. Stamper-Kurn, Phys. Rev. Lett. 98, 200801 (2007).

[38] S. Steinert, F. Dolde, P. Neumann, A. Aird, B. Naydenov, G. Balasubramanian, F. Jelezko, and J. Wrachtrup, Rev. Sci. Instrum. 81, 043705 (2010).

[39] J.P. Dowling, G. S. Agarwal, and W. P. Schleich, Phys. Rev. A 49, 4101 (1994). 